Journal of the Operations Research

Socicty of Japan

2008, Vol. 51, No. 2, 136-154

\title{
ON A LABOR ALLOCATION MODEL WITH POSSIBLY BOUNDED SALARIES
}

\author{
Rashid Farooq \\ National University of \\ Sciences and Technology
}

\author{
Yoshiko T Ikebe \\ Tokyo University of Science
}

Akihisa Tamura

Keio University

(Received September 29, 2005; Revised September 3, 2007)

\begin{abstract}
We consider pairwise stability in a labor allocation model with possibly bounded salaries, which is a common generalization of the marriage model and the assignment model. Our main contributions are results on complexity of checking pairwise stability and structures of pairwise stable outcomes.
\end{abstract}

Keywords: Algorithm, marriage model, assignment model, labor allocation, pairwise stability

\section{Introduction}

The theory of two-sided matching markets has seen many advances since the birth of the two standard models, the marriage model due to Gale and Shapley [4] and the assignment model of Shapley and Shubik [10]. The main progress has been the unification of these two standard models; Kaneko [5] formulated a general model using characteristic functions, and proved the nonemptiness of the core, Roth and Sotomayor [9] proposed a general model that also encompasses both and investigated the lattice property for payoffs, Eriksson and Karlander [1] proposed a hybrid model of the marriage model and the assignment model, and Fujishige and Tamura [2] proposed a generalization of the hybrid model due to Eriksson and Karlander [1] and Sotomayor [11] by utilizing $\mathrm{M}^{\natural}$-concave functions which play a central role in discrete convex analysis proposed by Murota [6-8]. Recently, Fujishige and Tamura [3] further generalized their model of [2] by adopting the idea of possibly bounded side payments. They showed the existence of a pairwise stable outcome but did not discuss their structures.

Our aim is to investigate these structures. However, the model of [3] is too general for analysis, so here we deal with a special case, which we call a labor allocation model with possibly bounded salaries. This model is a special case, but interesting in its own right, as it contains the assignment and marriage models as well as the Eriksson-Karlander model.

We consider two disjoint sets of agents, $P$ and $Q$, in a two-sided market where $P$ is a set of workers and $Q$ a set of firms. Each worker $i \in P$ can supply labor for several firms in $Q$, and each firm $j \in Q$ can employ several workers in $P$. If firm $j \in Q$ hires worker $i \in P$, worker $i$ receives a profit of $a(i, j)$ and firm $j$ a profit of $b(i, j)$. We assume in addition, that in this case $j$ pays a salary to $i$, which means that $i$ 's profit and $j$ 's profit respectively increase and decrease by the paid amount, and that each pair $(i, j) \in P \times Q$ has (agreed) lower and upper bounds on this salary. We will examine pairwise stability in this market.

The existence and characterization of pairwise stable outcomes will be derived from the results of [3] and described in Section 2. Our main contributions are in Sections 3 and 4, 
where we discuss the complexity of checking pairwise stability, and structures of pairwise stable outcomes.

\section{Characterization and Existence of Pairwise Stable Outcomes}

Mathematically, we describe our model in the following way. Let $E=P \times Q$ and define $E_{(i)}=\{i\} \times Q$ for $i \in P$ and $E_{(j)}=P \times\{j\}$ for $j \in Q$. For worker $i \in P, \lambda(i)$ denotes the maximum number of firms that $i$ can work for, and for firm $j \in Q, \mu(j)$ is the maximum number of workers that $j$ can hire. The profit vectors of workers and firms are denoted by $a=(a(i, j) \mid(i, j) \in E)$ and $b=(b(i, j) \mid(i, j) \in E)$, respectively. We assume that $\lambda=(\lambda(i) \mid i \in P) \in \mathbf{Z}_{+}^{P}, \mu=(\mu(j) \mid j \in Q) \in \mathbf{Z}_{+}^{Q}$ and $a=(a(i, j) \mid(i, j) \in E) \in \mathbf{R}^{E}, b=$ $(b(i, j) \mid(i, j) \in E) \in \mathbf{R}^{E}$, where $\mathbf{Z}_{+}^{P}\left(\mathbf{Z}_{+}^{Q}\right)$ denotes the nonnegative vectors of $\mathbf{Z}^{P}\left(\mathbf{Z}^{Q}\right)$. The lower and upper bounds of salaries are expressed by two vectors $\underline{\pi} \in(\mathbf{R} \cup\{-\infty\})^{E}$ and $\bar{\pi} \in(\mathbf{R} \cup\{+\infty\})^{E}$, which satisfy $\underline{\pi} \leq \bar{\pi}$. We represent labor allocation by a vector $x=(x(i, j) \mid(i, j) \in E) \in\{0,1\}^{E}$ where $x(i, j)$ is equal to one if $j$ hires $i$. For any $y \in \mathbf{R}^{E}$ and $k \in P \cup Q$, the restriction of $y$ on $E_{(k)}$ is denoted by $y_{(k)}$. For example, the vector $x_{(k)} \in\{0,1\}^{E_{(k)}}$ represents the labor allocation of agent $k \in P \cup Q$ with respect to $x \in\{0,1\}^{E}$.

A vector $x=(x(i, j) \mid(i, j) \in E) \in\{0,1\}^{E}$ is called a feasible allocation if $\sum_{k \in Q} x(i, k) \leq$ $\lambda(i)$ for all $i \in P$ and $\sum_{k \in P} x(k, j) \leq \mu(j)$ for all $j \in Q$. If a feasible allocation $x$ satisfies $\sum_{k \in Q} x(i, k)=\lambda(i)$, we say that $x$ saturates $i \in P$. Saturation for $j \in Q$ is similarly defined. A vector $s=(s(i, j) \mid(i, j) \in E) \in \mathbf{R}^{E}$ is called a feasible salary vector if $\underline{\pi} \leq s \leq \bar{\pi}$. An outcome is a pair $(x, s)$ consisting of a feasible allocation $x$ and a feasible salary vector $s$. For a given outcome $(x, s)$, let $X=\{(i, j) \in E \mid x(i, j)=1\}$. Then the pair $(x, s)$ is pairwise unstable if one of the following conditions holds:

(pu1) there exists $(i, j) \in X$ such that $a(i, j)+s(i, j)<0$ or $b(i, j)-s(i, j)<0$.

(pu2) there exist $(i, j) \in E \backslash X$ and $\alpha$ with $\underline{\pi}(i, j) \leq \alpha \leq \bar{\pi}(i, j)$ such that

(i) either $\left[a(i, j)+\alpha>0\right.$ and $\left.\sum_{k \in Q} x(i, k)<\lambda(i)\right]$ or $\left[\right.$ for some $\left(i, j^{\prime}\right) \in X$, $a(i, j)+\alpha>a\left(i, j^{\prime}\right)+s\left(i, j^{\prime}\right)$ and $\left.\sum_{k \in Q} x(i, k)=\lambda(i)\right]$

and

(ii) either $\left[b(i, j)-\alpha>0\right.$ and $\left.\sum_{k \in P} x(k, j)<\mu(j)\right]$ or $\left[\right.$ for some $\left(i^{\prime}, j\right) \in X$, $b(i, j)-\alpha>b\left(i^{\prime}, j\right)-s\left(i^{\prime}, j\right)$ and $\left.\sum_{k \in P} x(k, j)=\mu(j)\right]$.

Condition (pu1) implies that the hiring of worker $i$ by firm $j$ results in a negative profit for one side, which would of course wish to terminate the employer/employee relation, while condition (pu2) says that there exists a pair $(i, j)$ with no current employer/employee relation, with incentive to form one (possibly by terminating present relations). For example, if $i \in P$ is an agent unsaturated by $x$ with $a(i, j)+\alpha>0$ and $j \in Q$ is an agent saturated by $x$ satisfying the second condition in (ii), then $i$ would be motivated to work for $j$, and $j$ would hire $i$ instead of $i^{\prime}$ under salary $\alpha$. An outcome $(x, s)$ is pairwise stable if neither (pu1) nor (pu2) holds, or explicitly, if both of the following conditions are true:

(ps1) for all $(i, j) \in X, a(i, j)+s(i, j) \geq 0$ and $b(i, j)-s(i, j) \geq 0$.

(ps2) for all $(i, j) \in E \backslash X$ and $\alpha$ with $\underline{\pi}(i, j) \leq \alpha \leq \bar{\pi}(i, j)$,

$$
a(i, j)+\alpha \leq q_{i}^{(x, s)} \text { or } b(i, j)-\alpha \leq r_{j}^{(x, s)}
$$


where

$$
\begin{aligned}
q_{i}^{(x, s)} & = \begin{cases}\min \left\{a\left(i, j^{\prime}\right)+s\left(i, j^{\prime}\right) \mid\left(i, j^{\prime}\right) \in X\right\} & \text { if } i \text { is saturated by } x \\
0 & \text { otherwise, }\end{cases} \\
r_{j}^{(x, s)} & = \begin{cases}\min \left\{b\left(i^{\prime}, j\right)-s\left(i^{\prime}, j\right) \mid\left(i^{\prime}, j\right) \in X\right\} & \text { if } j \text { is saturated by } x \\
0 & \text { otherwise, }\end{cases}
\end{aligned}
$$

and the minimum over the empty set is understood to be $+\infty$. Note that if $\lambda=(1,1, \ldots, 1)$, $\mu=(1,1, \ldots, 1)$ and $\underline{\pi}=\bar{\pi}=(0,0, \ldots, 0)$ then pairwise stability is identical to stability in the marriage model. On the other hand, if $\lambda=(1,1, \ldots, 1), \mu=(1,1, \ldots, 1)$ and $\underline{\pi}=(-\infty,-\infty, \ldots,-\infty), \bar{\pi}=(+\infty,+\infty, \ldots,+\infty)$, then we have the assignment model. Thus our model is a common generalization of these two standard models.

Given sets $P, Q$ of agents and vectors $a, b, \underline{\pi}, \bar{\pi}, \lambda, \mu$, the first issue that comes to mind is the existence and a characterization of pairwise stable outcomes. The next theorem characterizes feasible allocations $x$ for which there exists a salary vector $p$ forming a pairwise stable outcome. It is a special case of Theorem 3.4 of [3], but here we give an independent proof.

Theorem 2.1. Let $x$ be a feasible allocation. There exists a feasible salary vector $s$ forming a pairwise stable outcome $(x, s)$ if and only if there exist a feasible salary vector $p \in \mathbf{R}^{E}$ and vectors $z_{P}, z_{Q} \in\{0,1\}^{E}$ such that

(as1) for all $i \in P, x_{(i)}$ is an optimal solution of

$$
\begin{array}{ll}
\text { maximize } & \sum_{k \in Q}(a(i, k)+p(i, k)) y(i, k) \\
\text { subject to } & \sum_{k \in Q} y(i, k) \leq \lambda(i), \\
& y(i, k) \leq z_{P}(i, k) \quad(k \in Q), \\
& y(i, k) \in\{0,1\} \quad(k \in Q) .
\end{array}
$$

(as2) for all $j \in Q, x_{(j)}$ is an optimal solution of

$$
\begin{array}{ll}
\text { maximize } & \sum_{k \in P}(b(k, j)-p(k, j)) y(k, j) \\
\text { subject to } & \sum_{k \in P} y(k, j) \leq \mu(j), \\
& y(k, j) \leq z_{Q}(k, j) \quad(k \in P), \\
& y(k, j) \in\{0,1\} \quad(k \in P) .
\end{array}
$$

(as3) $z_{P}(i, j)=0 \Rightarrow p(i, j)=\underline{\pi}(i, j), z_{Q}(i, j)=1$.

(as4) $z_{Q}(i, j)=0 \Rightarrow p(i, j)=\bar{\pi}(i, j), z_{P}(i, j)=1$.

Intuitively, the pairs $(i, j)$ for which $z_{P}(i, j)=0$ are the pairs which are attractive to agent $i \in P$ but not to agent $j \in Q$, in the sense that even with lowest possible salary of $\underline{\pi}(i, j), i$ would be inclined to work for $j$, but $j$ would not be motivated to hire $i$ (and likewise for $z_{Q}$ ).

Proof. $(\Leftarrow) \quad$ In this case we show that $(x, p)$ is a pairwise stable outcome. Let $X=\{(i, j) \in$ $E \mid x(i, j)=1\}$. From (as1),$(i, j) \in X$, i.e. $x(i, j)=1$ implies $a(i, j)+p(i, j) \geq 0$. Similarly, we have $b(i, j)-p(i, j) \geq 0$ for $(i, j) \in X$. Hence (pu1) does not hold.

Now let $(i, j) \in E \backslash X$. Because $x(i, j)=0$, the optimality of $x_{(i)}$ implies that when $z_{P}(i, j)=1$, one of (a1) and (a2) is true:

$$
\begin{array}{ll}
\text { (a1) : } & \sum_{k \in Q} x(i, k)<\lambda(i) \text { and } a(i, j)+p(i, j) \leq 0, \\
\text { (a2) : } & \sum_{k \in Q} x(i, k)=\lambda(i) \text { and } \\
& a(i, j)+p(i, j) \leq a\left(i, j^{\prime}\right)+p\left(i, j^{\prime}\right) \text { for all } j^{\prime} \in Q \text { with } x\left(i, j^{\prime}\right)=1 .
\end{array}
$$


Taking the case $z_{P}(i, j)=0$ into account, and applying (as3), we can see that at least one of $(\mathrm{a} 0)$ to $(\mathrm{a} 2)$ must hold:

$$
\text { (a0): } z_{P}(i, j)=0 \text {, hence } z_{Q}(i, j)=1 \text { and } p(i, j)=\underline{\pi}(i, j)
$$

Similarly, at least one of (b0) to (b2) must hold:

(b0): $z_{Q}(i, j)=0$, hence $z_{P}(i, j)=1$ and $p(i, j)=\bar{\pi}(i, j)$,

(b1): $\quad \sum_{k \in P} x(k, j)<\mu(j)$ and $b(i, j)-p(i, j) \leq 0$,

(b2) : $\quad \sum_{k \in P} x(k, j)=\mu(j)$ and

$b(i, j)-p(i, j) \leq b\left(i^{\prime}, j\right)-p\left(i^{\prime}, j\right)$ for all $i^{\prime} \in P$ with $x\left(i^{\prime}, j\right)=1$.

Note that

$$
\begin{aligned}
& (\mathrm{a} 0) \Rightarrow b(i, j)-\alpha \leq b(i, j)-p(i, j) \text { for all } \alpha \text { with } \underline{\pi}(i, j) \leq \alpha \leq \bar{\pi}(i, j) \\
& (\mathrm{b} 0) \Rightarrow a(i, j)+\alpha \leq a(i, j)+p(i, j) \text { for all } \alpha \text { with } \underline{\pi}(i, j) \leq \alpha \leq \bar{\pi}(i, j)
\end{aligned}
$$

and that (a0) and (b0) cannot be simultaneously true. We can now consider in turn the eight cases, at least one of which must hold.

Case (a0) and (b1): When this occurs, by the preceding observation on (a0), we have that $\overline{\sum_{k \in P} x(k, j)<\mu(j)}$ and $b(i, j)-\alpha \leq 0$ for all $\alpha$ with $\underline{\pi}(i, j) \leq \alpha \leq \bar{\pi}(i, j)$, and (pu2) cannot hold.

Case (a0) and (b2): We may immediately conclude that $\sum_{k \in P} x(k, j)=\mu(j)$ and $b(i, j)-$ $\overline{\alpha \leq b\left(i^{\prime}, j\right)-p\left(i^{\prime}, j\right)}$ for all $\left(i^{\prime}, j\right) \in X$, and (pu2) cannot be true.

Case (a1) and (b1): Here we have $\sum_{k \in Q} x(i, k)<\lambda(i)$ and $\sum_{k \in P} x(k, j)<\mu(j)$. If (pu2) were true, we would have $a(i, j)+\alpha>0$ and $b(i, j)-\alpha>0$ for some $\alpha$, which implies that $a(i, j)+b(i, j)>0$. However, since $a(i, j)+b(i, j)=(a(i, j)+p(i, j))+(b(i, j)-p(i, j)) \leq 0$, this cannot occur.

Case (a1) and (b2): We must have $\sum_{k \in Q} x(i, k)<\lambda(i)$ and $\sum_{k \in P} x(k, j)=\mu(j)$. As in the previous case, for (pu2) to hold, we need $a(i, j)+b(i, j)>b\left(i^{\prime}, j\right)-p\left(i^{\prime}, j\right)$ for some $\left(i^{\prime}, j\right) \in X$, however (a1) and (b2) imply the converse.

Case (a2) and (b2): In this case, $\sum_{k \in Q} x(i, k)=\lambda(i)$ and $\sum_{k \in P} x(k, j)=\mu(j)$ must hold.

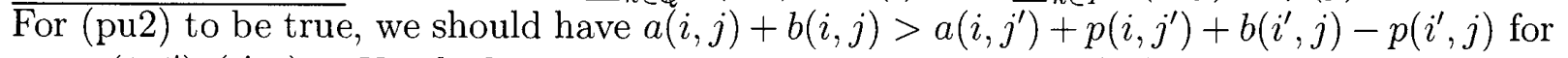
some $\left(i, j^{\prime}\right),\left(i^{\prime}, j\right) \in X$, which is contrary to the implications of (a2) and (b2).

The remaining cases can be proved analogously.

$(\Rightarrow)$ Let $(x, s)$ be a pairwise stable outcome. We will define $p, z_{P}$ and $z_{Q}$ satisfying (as1) to (as4).

If $(i, j) \in E$ satisfies $x(i, j)=1$, simply set $z_{P}(i, j)=z_{Q}(i, j)=1$ and $p(i, j)=s(i, j)$.

Now consider $(i, j) \in E$ with $x(i, j)=0$. Define $z_{P}(i, j)$ and $z_{Q}(i, j)$ as follows:

$$
\begin{aligned}
& z_{P}(i, j)= \begin{cases}0 & \text { if } a(i, j)+\underline{\pi}(i, j)>q_{i}^{(x, s)} \\
1 & \text { otherwise }\end{cases} \\
& z_{Q}(i, j)= \begin{cases}0 & \text { if } b(i, j)-\bar{\pi}(i, j)>r_{j}^{(x, s)} \\
1 & \text { otherwise }\end{cases}
\end{aligned}
$$

where $q_{i}^{(x, s)}$ and $r_{j}^{(x, s)}$ are the values given by (2.1) and (2.2). Because $(x, s)$ is a pairwise stable outcome, it is easy to see that $z_{P}(i, j)$ and $z_{Q}(i, j)$ cannot simultaneously be 0 . 
We now define the vector $p$. If $z_{P}(i, j)=0$ then set $p(i, j)=\underline{\pi}(i, j)$. Likewise, if $z_{Q}(i, j)=$ 0 then set $p(i, j)=\bar{\pi}(i, j)$. When $z_{P}(i, j)=z_{Q}(i, j)=1$, then we see from the pairwise stability of $(x, s)$ that

$$
\begin{aligned}
& a(i, j)+\alpha \leq q_{i}^{(x, s)} \text { or } b(i, j)-\alpha \leq r_{j}^{(x, s)} \quad \text { for all } \alpha \in[\underline{\pi}(i, j), \bar{\pi}(i, j)] \\
& a(i, j)+\underline{\pi}(i, j) \leq q_{i}^{(x, s)} \text { and } b(i, j)-\bar{\pi}(i, j) \leq r_{j}^{(x, s)}
\end{aligned}
$$

It is easy to see that there always exists an $\alpha \in[\underline{\pi}(i, j), \bar{\pi}(i, j)]$ satisfying

$$
a(i, j)+\alpha \leq q_{i}^{(x, s)} \text { and } b(i, j)-\alpha \leq r_{j}^{(x, s)}
$$

so let $p(i, j)$ be any such $\alpha$.

For these $z_{P}, z_{Q}$ and $p$, (as3) and (as4) obviously hold. Moreover, by the pairwise stability of $(x, s)$, if $z_{Q}(i, j)=0$ then we must have

$$
\begin{aligned}
& \sum_{k \in Q} x(i, k)<\lambda(i) \quad \Rightarrow a(i, j)+\bar{\pi}(i, j) \leq 0, \\
& \sum_{k \in Q} x(i, k)=\lambda(i) \Rightarrow a(i, j)+\bar{\pi}(i, j) \leq q_{i}^{(x, s)} .
\end{aligned}
$$

Hence, for any fixed $i$, we have $a(i, j)+p(i, j) \geq a\left(i, j^{\prime}\right)+p\left(i, j^{\prime}\right)$ for any $j, j^{\prime}$ with $x(i, j)=1$ and $x\left(i, j^{\prime}\right)=0, z_{P}\left(i, j^{\prime}\right)=1$, which guarantees (as1). The proof of (as2) is analogous.

The existence of a pairwise outcome is a direct consequence of Theorem 3.3 of [3].

Theorem 2.2. For any instance, a pairwise stable outcome always exists.

\section{Complexity of Checking Pairwise Stability}

In this section, we deal with the problem where we are given only a feasible allocation or salary vector, and we wish to decide whether it can be "expanded" into a pairwise stable outcome. We will say that a feasible allocation $x$ is pairwise stable if there exists a feasible salary vector $s$ such that $(x, s)$ is a pairwise stable outcome, otherwise it is pairwise unstable. Pairwise stable and unstable salary vectors are defined similarly. With this terminology, Theorem 2.1 of the preceding section may be seen as one characterization of pairwise stable allocations. In the next subsection, we show that checking pairwise stability of feasible allocations may be accomplished in polynomial time.

\subsection{Checking pairwise stability of a feasible allocation}

First, we give two propositions which will be used in the proof.

Proposition 3.1. Given numbers $q_{i}$ and $r_{j}$, we have

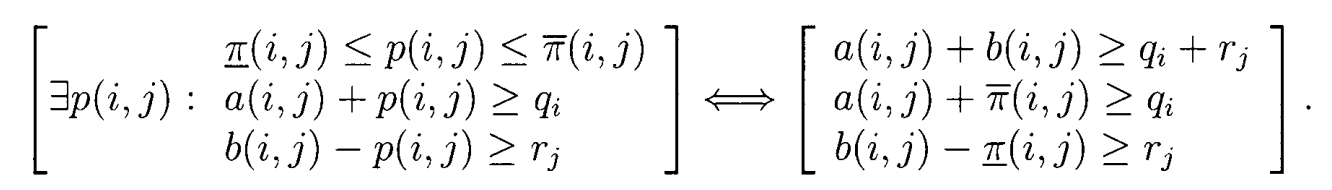

Proof. We show the direction $(\Leftarrow)$. If $\underline{\pi}(i, j) \leq q_{i}-a(i, j)$, then by setting $p(i, j):=$ $q_{i}-a(i, j)$, we have

$$
\begin{aligned}
& \underline{\pi}(i, j) \leq q_{i}-a(i, j)=p(i, j) \\
& a(i, j)+p(i, j)=q_{i} \\
& b(i, j)-p(i, j)=b(i, j)+a(i, j)-q_{i} \geq r_{j} \\
& \bar{\pi}(i, j)-p(i, j)=\bar{\pi}(i, j)+a(i, j)-q_{i} \geq 0
\end{aligned}
$$


If $q_{i}-a(i, j) \leq \underline{\pi}(i, j)$, then by setting $p(i, j):=\underline{\pi}(i, j)$, we have

$$
\begin{aligned}
& \underline{\pi}(i, j)=p(i, j) \leq \bar{\pi}(i, j), \\
& a(i, j)+p(i, j)=a(i, j)+\underline{\pi}(i, j) \geq q_{i}, \\
& b(i, j)-p(i, j)=b(i, j)-\underline{\pi}(i, j) \geq r_{j} .
\end{aligned}
$$

The direction $(\Rightarrow)$ is trivial.

Proposition 3.2. Given numbers $q_{i}$ and $r_{j}$, we have

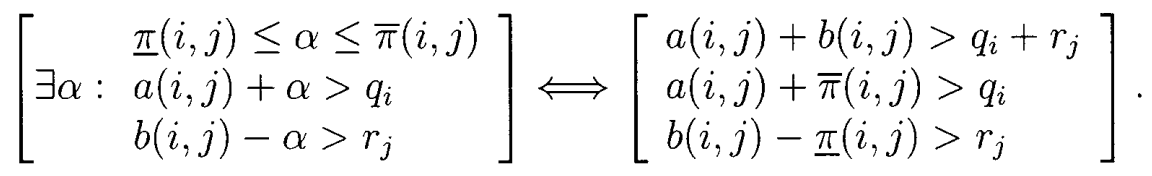

Proof. We can show the assertion in the same way as the proof of Proposition 3.1. To show the direction $(\Leftarrow)$, it is enough to set $\alpha:=q_{i}-a(i, j)+\varepsilon$ for a sufficiently small positive number $\varepsilon$ if $\underline{\pi}(i, j) \leq q_{i}-a(i, j)$; otherwise $\alpha:=\underline{\pi}(i, j)$.

We note that Propositions 3.1 and 3.2 guarantee that the condition (ps2) for a given feasible outcome $(x, s)$ can be checked in $O(|E|)$ time, thus, the pairwise stability of a given outcome can be checked in $O(|E|)$ time.

We now describe our algorithm Allocation_Stability (which is given at the end of this subsection) for deciding the pairwise stability of a given feasible allocation. We fix a feasible allocation $x$, and assume that $x$ is pairwise stable. With this assumption, we attempt to construct a feasible salary vector $s$ such that $(x, s)$ is pairwise stable. If $x$ is indeed pairwise stable, such an $s$ will always be found. If it is not, then the procedure will encounter a contradiction, thus discover a proof of pairwise unstability.

Let $X:=\{(i, j) \in E \mid x(i, j)=1\}$. We initially define by $E \backslash X$ the set $B$, which is the set of candidates which may break the pairwise stability of $x$. When an element of $B$ is established not to hinder pairwise stability of $x$, it will be eliminated.

If $b(i, j)<\underline{\pi}(i, j)$ for some $(i, j) \in X$ then $x$ is trivially pairwise unstable. From now on, we assume that $b(i, j) \geq \underline{\pi}(i, j)$ for all $(i, j) \in X$.

We first construct $q=\left(q_{i}: i \in P\right) \in \mathbf{R}^{P}$ such that

$$
\begin{aligned}
& q_{i} \leq a(i, j)+s(i, j) \quad(\forall(i, j) \in X) \\
& \text { for all } s \text { such that }(x, s) \text { is pairwise stable. }
\end{aligned}
$$

(If $x$ is pairwise unstable, any $q$ satisfies (3.1).) Since $q=\mathbf{0}$ satisfies (3.1), we initially set $q:=0$. Then, we slightly update $q$ by the following reasoning: for some $(i, j) \in B$, if $j$ is unsaturated by $x, b(i, j)-\bar{\pi}(i, j)>0$ and $a(i, j)+\bar{\pi}(i, j)>0$ then $a\left(i, j^{\prime}\right)+s\left(i, j^{\prime}\right) \geq$ $a(i, j)+\bar{\pi}(i, j)\left(\forall\left(i, j^{\prime}\right) \in X\right)$ for all $s$ for which $(x, s)$ is pairwise stable; since otherwise, $(i, j)$ and $\alpha=\bar{\pi}(i, j)$ satisfy (pu2) for $(x, s)$. Hence we update $q_{i}$ as

$$
q_{i}:=\max \left\{\begin{array}{l|l}
0, \max \{a(i, j)+\bar{\pi}(i, j) & \begin{array}{l}
(i, j) \in B, b(i, j)-\bar{\pi}(i, j)>0 \\
\text { and } j \text { is unsaturated by } x
\end{array}
\end{array}\right\} .
$$

Set

$$
p_{i j}:=\max \left\{q_{i}-a(i, j), \underline{\pi}(i, j)\right\}
$$

for all $(i, j) \in X$ and define

$$
r_{j}:=\left\{\begin{array}{cl}
\min \left\{b(i, j)-p_{i j} \mid(i, j) \in X\right\} & \text { if } j \text { is saturated by } x \\
0 & \text { otherwise }
\end{array}\right.
$$


for all $j \in Q$. For a feasible salary vector $s$ such that $(x, s)$ is pairwise stable, $(3.1)$ and (3.3) imply $p_{i j} \leq s(i, j)$ for all $(i, j) \in X$. This says that (3.1) induces

$$
\begin{array}{ll}
r_{j} \geq \min \{b(i, j)-s(i, j) \mid(i, j) \in X\} & (\forall j \text { saturated by } x) \\
r_{j}=0 & (\forall j \text { unsaturated by } x) \\
\text { for all } s \text { such that }(x, s) \text { is pairwise stable. } &
\end{array}
$$

The above condition (3.5) will be maintained as an invariant during the execution of our algorithm. The $r$-values, which will be nonincreasing, will be updated in such a way that they always satisfy (3.5). The $q$-values, which will be nondecreasing, in their turn will always satisfy the next condition as well as (3.1):

at the point when the update of $q_{i}$ is completed, for some $(i, j) \in B$ and some $r$ satisfying (3.5), either $\left[q_{i}=a(i, j)+\bar{\pi}(i, j)\right.$ and $\left.b(i, j)-\bar{\pi}(i, j)>r_{j}\right]$ or $\left[q_{i}=a(i, j)+b(i, j)-r_{j}\right.$ and $\left.\underline{\pi}(i, j)<b(i, j)-r_{j} \leq \bar{\pi}(i, j)\right]$.

Note that when $q_{i}$ is strictly increased from 0 by (3.2), only the first case occurs.

We assume that $q$ and $r$ satisfying (3.1), (3.5), and (3.6) are given. In addition, we assume that $r$ is calculated by (3.3) and (3.4) for the current $q$. The following claim provides a certificate of pairwise unstability.

Claim 3.3. For some $(i, j) \in X$, if either (1) $i$ is unsaturated by $x$ and $q_{i}>0$, or (2) $q_{i}>a(i, j)+\bar{\pi}(i, j)$, or $(3) q_{i}>a(i, j)+b(i, j)$, then $x$ is pairwise unstable.

Proof. We show the contraposition, and hence, assume that $(x, s)$ is a pairwise stable outcome. Let $(i, j)$ be any element of $X$. By (3.1), we have $q_{i} \leq a(i, j)+s(i, j) \leq a(i, j)+\bar{\pi}(i, j)$, the negation of (2). By combining the above inequality with $0 \leq b(i, j)-s(i, j)$ which is obtained by the pairwise stability of $(x, s)$, we have $q_{i} \leq a(i, j)+b(i, j)$, the negation of (3). Finally consider (1). Suppose to the contrary that $i$ is unsaturated by $x$ and $q_{i}>0$. By (3.6), either $q_{i}=a(i, k)+\bar{\pi}(i, k)>0$ or $q_{i}=a(i, k)+b(i, k)-r_{k}>0$ for some $(i, k) \in B$. By setting $\alpha:=\bar{\pi}(i, k)$ in the former case and $\alpha:=b(i, k)-r_{k}-\varepsilon$ in the latter case for a sufficiently small positive number $\varepsilon$, we have $\underline{\pi}(i, k) \leq \alpha \leq \bar{\pi}(i, k), a(i, k)+\alpha>0=q_{i}^{(x, s)}$ and $b(i, k)-\alpha>r_{k} \geq r_{k}^{(x, s)}$ which contradict the pairwise stability of $(x, s)$.

The following claims provide a proof of pairwise stability.

Claim 3.4. Assume that none of the conditions of Claim 3.3 holds, and that $r$ was calculated by (3.3) and (3.4). For all $(i, j) \in X$, we have

$$
a(i, j)+b(i, j) \geq q_{i}+r_{j}, \quad a(i, j)+\bar{\pi}(i, j) \geq q_{i}, \text { and } \quad b(i, j)-\underline{\pi}(i, j) \geq r_{j} \geq 0 .
$$

Proof. Since no condition in Claim 3.3 holds, we have $a(i, j)+\bar{\pi}(i, j) \geq q_{i}$. We suppose that $j$ is saturated by $x$. By (3.3) and (3.4), we have the first and last inequalities as follows:

$$
\begin{gathered}
b(i, j)-\left(q_{i}-a(i, j)\right) \geq b(i, j)-p_{i j} \geq r_{j}, \\
b(i, j)-\underline{\pi}(i, j) \geq b(i, j)-p_{i j} \geq r_{j} .
\end{gathered}
$$

The nonnegativity of $r_{j}$ follows from $a(i, j)+b(i, j) \geq q_{i}, b(i, j) \geq \underline{\pi}(i, j)$ for all $(i, j) \in X$, and the fact that $r$ was defined by (3.3) and (3.4). We next suppose that $j$ is unsaturated by $x$, i.e., $r_{j}=0$ holds by (3.4). The assumptions that $a(i, j)+b(i, j) \geq q_{i}$ and $b(i, j) \geq \underline{\pi}(i, j)$ show the first and last inequalities. 
Claim 3.5. If a pair $(i, j) \in E \backslash X$ satisfies $a(i, j)+b(i, j) \leq q_{i}+r_{j}$, or $a(i, j)+\bar{\pi}(i, j) \leq q_{i}$, or $b(i, j)-\underline{\pi}(i, j) \leq r_{j}$, then there exists no feasible $\alpha$ such that $a(i, j)+\alpha>q_{i}$ and $b(i, j)-\alpha>r_{j}$. Thus, under the same assumptions as Claim 3.4, if every pair $(i, j)$ of $E \backslash X$ satisfies one of the three inequalities stated above, then $x$ is pairwise stable.

Proof. The first assertion follows from Proposition 3.2. By Claim 3.4 and Proposition 3.1, for each $(i, j) \in X$, there exists a feasible $s(i, j)$ such that $a(i, j)+s(i, j) \geq q_{i} \geq 0$ and $b(i, j)-s(i, j) \geq r_{j} \geq 0$. By appropriately defining $s(i, j)$ for all $(i, j) \in E \backslash X$, we obtain a pairwise stable outcome $(x, s)$.

Next suppose that neither pairwise unstability nor stability has been established. We may further assume that

$$
b(i, j)-\underline{\pi}(i, j) \geq r_{j} \quad \text { for all }(i, j) \in X
$$

because (3.7) holds after a calculation of $r$ by (3.3) and (3.4), and because once it is satisfied, it will thereafter be preserved under the assumption that $r$ is nonincreasing. Note that checking the conditions of Claims 3.3 and 3.5 does not involve modification to $q$ or $r$, thus the invariants (3.1), (3.5) and (3.6) are retained. In this case, we update $q$ and $r$ values in such a way that the number of arcs $(i, j) \in E \backslash X$ which satisfy one of three conditions of Claim 3.5 increases (or pairwise unstability is verified). Of the three possible conditions of Claim 3.5, $a(i, j)+\bar{\pi}(i, j) \leq q_{i}$ is monotonic in the sense that once it is satisfied for $(i, j) \in E \backslash X$, it will thereafter be preserved, since $q_{i}$ is nondecreasing. When we interpret $B$, which is initially defined by $E \backslash X$, as the set of edges for which the conditions of Claim 3.5 must be checked, such $(i, j)$ may be eliminated. Hereafter, we assume that $a(i, j)+\bar{\pi}(i, j)>q_{i}$ holds for each $(i, j) \in B$, and for some $(i, j) \in B$, both $a(i, j)+b(i, j)>q_{i}+r_{j}$ and $b(i, j)-\underline{\pi}(i, j)>r_{j}$ are satisfied. Let us consider a nonempty subset $\widetilde{B}$ of $B$ defined by

$$
\widetilde{B}:=\left\{(i, j) \in B \mid b(i, j)-\underline{\pi}(i, j)>r_{j}\right\} .
$$

Since $r$ does not increase in our algorithm, no element of $\widetilde{B}$ is deleted from $\widetilde{B}$ except when it is eliminated from $B$. Let $\widetilde{G}=(P, Q ; X \cup \widetilde{B})$ be the directed graph in which each pair in $X$ is oriented from $P$-vertex to $Q$-vertex, and each pair in $\widetilde{B}$ is oriented from $Q$-vertex to $P$-vertex. (However, to avoid confusion, we will consider an element of $\widetilde{B}$ as a pair belonging to $P \times Q$, and the notation $(u, v) \in \widetilde{B}$ will mean $u \in P$ and $v \in Q$.) We also define arc lengths $\ell: X \cup \widetilde{B} \rightarrow \mathbf{R}$ by

$$
\ell(u, v):= \begin{cases}a(u, v)+b(u, v)-q_{u}-r_{v} & \text { if }(u, v) \in X \\ q_{v}+r_{u}-a(v, u)-b(v, u) & \text { if }(v, u) \in \widetilde{B}\end{cases}
$$

By Claim 3.4, the length of each arc associated with $X$ is nonnegative. We say that a directed cycle $C$ in $\widetilde{G}$ is a negative cycle if the amount $\ell(C)$ defined as the sum of the lengths of all arcs in $C$ is negative. The next proposition will help us to update $q$ and $r$ when $\widetilde{G}$ has a negative cycle.

Proposition 3.6. Let $C$ be a negative cycle. Then we may choose a vertex $v_{0}$ so that $C=v_{0}, v_{1}, \ldots, v_{k}\left(=v_{0}\right)$, and all paths $v_{0}, v_{1}, \ldots, v_{i}(1 \leq i \leq k)$ have negative weight.

Proof. If all arcs of $C$ have nonpositive weight, then the claim is trivially true. So assume that at least one arc has positive weight. Consider all sequences of consecutive $\operatorname{arcs}$ of $C$, and choose one with maximum (positive) weight. If there is more than one such sequence, select 
a longest one. Name the vertices of this sequence as $u_{0}, u_{1}, \ldots, u_{j}$, and the other vertices of $C$ as $u_{j+1}, \ldots, u_{k}\left(=u_{0}\right)$. We will show that the end vertex of the maximum weight sequence, $u_{j}$, satisfies the properties of the assertion.

First we note that all paths $u_{j}, u_{j+1}, \ldots, u_{l}(j+1 \leq l \leq k)$ must have negative weight, for otherwise it would contradict the fact that $u_{0}, u_{1}, \ldots, u_{j}$ was chosen as the longest sequence of maximum weight. Now, suppose that for some $l(1 \leq l \leq j-1)$, the path $u_{j}, u_{j+1}, \ldots, u_{0}, u_{1}, \ldots, u_{l}$ has nonnegative weight. Then this implies that $u_{l}, u_{l+1}, \ldots, u_{j}$ is a negative weight sequence, again contradicting the choice of $u_{0}, \ldots, u_{j}$ as a maximum weight sequence. Thus, the sequence $u_{j}, u_{j+1}, \ldots, u_{0}, u_{1}, \ldots, u_{l}$ also has negative weight.

From now on, we divide our argument into two cases according to the existence of a negative cycle in $\widetilde{G}$.

We first suppose that $\widetilde{G}$ has a negative cycle, which must have an even number of vertices because $\widetilde{G}$ is bipartite. Let $C=v_{0}, v_{1}, \ldots, v_{2 k}\left(=v_{0}\right)$ be a negative cycle satisfying the condition of Proposition 3.6. We have $\left(v_{1}, v_{0}\right) \in \widetilde{B}$ because $\ell\left(v_{0}, v_{1}\right)<0$. Since $b\left(v_{1}, v_{0}\right)-$ $\underline{\pi}\left(v_{1}, v_{0}\right)>r_{v_{0}}$ and $r$ does not increase in our algorithm, if $x$ is indeed pairwise stable then we can update $q_{v_{1}}$ as $\min \left\{a\left(v_{1}, v_{0}\right)+b\left(v_{1}, v_{0}\right)-r_{v_{0}}, a\left(v_{1}, v_{0}\right)+\bar{\pi}\left(v_{1}, v_{0}\right)\right\}$, because for every sufficiently small positive number $\varepsilon, \alpha=\min \left\{b\left(v_{1}, v_{0}\right)-r_{v_{0}}-\varepsilon, \bar{\pi}\left(v_{1}, v_{0}\right)\right\}$ satisfies $\underline{\pi}\left(v_{1}, v_{0}\right) \leq \alpha \leq \bar{\pi}\left(v_{1}, v_{0}\right)$ and $b\left(v_{1}, v_{0}\right)-\alpha \geq r_{v_{0}}+\varepsilon>r_{v_{0}}$, and because (3.5) implies $a\left(v_{1}, j\right)+s\left(v_{1}, j\right) \geq a\left(v_{1}, v_{0}\right)+\alpha$ for all $\left(v_{1}, j\right) \in X$ and for all feasible salary vectors $s$ such that $(x, s)$ are pairwise stable. Thus, set

$$
\text { (new) } q_{v_{1}}:=\min \left\{a\left(v_{1}, v_{0}\right)+b\left(v_{1}, v_{0}\right)-r_{v_{0}}, a\left(v_{1}, v_{0}\right)+\bar{\pi}\left(v_{1}, v_{0}\right)\right\}
$$

where $($ new $) *$ denotes a value after the update. We note that $q_{v_{1}}$ strictly increases and the above update preserves the invariants (3.1) and (3.6). If $v_{1}$ is unsaturated by $x$ then $x$ must be pairwise unstable by (1) of Claim 3.3. Furthermore, if (new) $q_{v_{1}}=a\left(v_{1}, v_{0}\right)+\bar{\pi}\left(v_{1}, v_{0}\right)$ then we can eliminate $\left(v_{1}, v_{0}\right)$ from $B$ (see Claim 3.5). Thus we assume that $v_{1}$ is saturated by $x$ and (new) $q_{v_{1}}<a\left(v_{1}, v_{0}\right)+\bar{\pi}\left(v_{1}, v_{0}\right)$, which says that $q_{v_{1}}$ was increased by $-\ell\left(v_{0}, v_{1}\right)$. Since $\widetilde{G}$ is bipartite, we have $\left(v_{1}, v_{2}\right) \in X$. By (3) of Claim 3.3, if (new) $q_{v_{1}}>a\left(v_{1}, v_{2}\right)+b\left(v_{1}, v_{2}\right)$ then $x$ must be pairwise unstable. We suppose that (new) $q_{v_{1}} \leq a\left(v_{1}, v_{2}\right)+b\left(v_{1}, v_{2}\right)$, and set

$$
\text { (new) } r_{v_{2}}:=a\left(v_{1}, v_{2}\right)+b\left(v_{1}, v_{2}\right)-(\text { new }) q_{v_{1}} \geq 0 \text {. }
$$

Then we can show that $v_{2}$ is saturated by $x$, (new) $q_{v_{1}}-a\left(v_{1}, v_{2}\right)>\underline{\pi}\left(v_{1}, v_{2}\right)$, and (new) $r_{v_{2}}<$ (old) $r_{v_{2}}$ by

$$
\begin{aligned}
0 & >\ell\left(v_{0}, v_{1}\right)+\ell\left(v_{1}, v_{2}\right) \\
& =\text { (old }) q_{v_{1}}-(\text { new }) q_{v_{1}}+a\left(v_{1}, v_{2}\right)+b\left(v_{1}, v_{2}\right)-(\text { old }) q_{v_{1}}-(\text { old }) r_{v_{2}} \\
& =a\left(v_{1}, v_{2}\right)+b\left(v_{1}, v_{2}\right)-(\text { new }) q_{v_{1}}-(\text { old }) r_{v_{2}} \\
& \geq a\left(v_{1}, v_{2}\right)-(\text { new }) q_{v_{1}}+\underline{\pi}\left(v_{1}, v_{2}\right)
\end{aligned}
$$

where (old)* denotes values before the update, and the first and last inequalities follow from Proposition 3.6 and (3.7), respectively. Note that if $v_{2}$ were unsaturated by $x$, the above inequality would imply (new) $q_{v_{1}}>a\left(v_{1}, v_{2}\right)+b\left(v_{1}, v_{2}\right)$ which contradicts the assumption. Let $p_{v_{1} v_{2}}:=($ new $) q_{v_{1}}-a\left(v_{1}, v_{2}\right)$. For a feasible salary vector $s$ such that $(x, s)$ is pairwise stable, (3.1) and the definition of $p_{v_{1} v_{2}}$ imply $\underline{\pi}\left(v_{1}, v_{2}\right) \leq p_{v_{1} v_{2}} \leq s\left(v_{1}, v_{2}\right)$. Thus, the above update of $r_{v_{2}}\left(=b\left(v_{1}, v_{2}\right)-p_{v_{1} v_{2}}\right)$ preserves the invariant (3.5). The above calculation also 
says that $r_{v_{2}}$ is decreased by $-\ell\left(v_{0}, v_{1}\right)-\ell\left(v_{1}, v_{2}\right)$. Similarly we modify $q_{v_{2 i+1}}$ and $r_{v_{2 i+2}}$ for $i=1, \ldots, k-1$ by the following procedure:

$$
\begin{aligned}
& \text { for } i:=0 \text { to } k-1 \text { do }\{ \\
& q_{v_{2 i+1}}:=\min \left\{a\left(v_{2 i+1}, v_{2 i}\right)+b\left(v_{2 i+1}, v_{2 i}\right)-r_{v_{2 i}}, a\left(v_{2 i+1}, v_{2 i}\right)+\bar{\pi}\left(v_{2 i+1}, v_{2 i}\right)\right\} \\
& \text { if either } \\
& \quad(1) q_{v_{2 i+1}}=a\left(v_{2 i+1}, v_{2 i}\right)+\bar{\pi}\left(v_{2 i+1}, v_{2 i}\right) \text {, or } \\
& \quad(2) v_{2 i+1} \text { is unsaturated by } x, \text { or } \\
& \quad \text { (3) } q_{v_{2 i+1}}>a\left(v_{2 i+1}, v_{2 i+2}\right)+b\left(v_{2 i+1}, v_{2 i+2}\right) \\
& \text { then go to Step } 1 \text { of ALLOCATION_STABILITY; } \\
& r_{v_{2 i+2}}:=a\left(v_{2 i+1}, v_{2 i+2}\right)+b\left(v_{2 i+1}, v_{2 i+2}\right)-q_{v_{2 i+1}} \\
& \}
\end{aligned}
$$

Claim 3.7. At each iteration $i$ in the above procedure, we have

(a) the invariants (3.1) and (3.6) are preserved,

(b) if the process does not go to Step 1 of Allocation_Stability, then $q_{v_{2 i+1}}$ is increased by $-\sum_{j=0}^{i-1}\left[\ell\left(v_{2 j}, v_{2 j+1}\right)+\ell\left(v_{2 j+1}, v_{2 j+2}\right)\right]-\ell\left(v_{2 i}, v_{2 i+1}\right)$,

(c) if the process does not go to Step 1 of AllocATION_STABILITY, then $v_{2 i+2}$ is saturated by $x, r_{v_{2 i+2}}$ is decreased by $-\sum_{j=0}^{i}\left[\ell\left(v_{2 j}, v_{2 j+1}\right)+\ell\left(v_{2 j+1}, v_{2 j+2}\right)\right]$, and the invariant (3.5) is preserved,

(d) when the process goes to Step 1 of ALlocation_STABILITY, if (1) holds then at least one pair can be eliminated from $B$; otherwise $x$ is pairwise unstable.

Proof. We have already proved the above assertions for the first iteration (i.e., for $i=0$ ). We consider iteration $i$ with $i \geq 1$ and assume that the assertions hold for the previous iterations. Assertion (a) can be shown in the same way as in the first iteration. We assume that the process does not go to Step 1 of Allocation_Stability, and show (b) and (c). In this case, by the induction hypothesis, we have

$$
\begin{aligned}
(\text { new }) q_{v_{2 i+1}}-(\text { old }) q_{v_{2 i+1}}= & a\left(v_{2 i+1}, v_{2 i}\right)+b\left(v_{2 i+1}, v_{2 i}\right)-(\text { new }) r_{v_{2 i}}-(\text { old }) q_{v_{2 i+1}} \\
= & a\left(v_{2 i+1}, v_{2 i}\right)+b\left(v_{2 i+1}, v_{2 i}\right)-(\text { old }) q_{v_{2 i+1}}-(\text { old }) r_{v_{2 i}} \\
& -\sum_{j=0}^{i-1}\left[\ell\left(v_{2 j}, v_{2 j+1}\right)+\ell\left(v_{2 j+1}, v_{2 j+2}\right)\right] \\
= & -\ell\left(v_{2 i}, v_{2 i+1}\right)-\sum_{j=0}^{i-1}\left[\ell\left(v_{2 j}, v_{2 j+1}\right)+\ell\left(v_{2 j+1}, v_{2 j+2}\right)\right]
\end{aligned}
$$

that is, we have (b). Similarly, we have

$$
\begin{aligned}
0 & >\sum_{j=0}^{i}\left[\ell\left(v_{2 j}, v_{2 j+1}\right)+\ell\left(v_{2 j+1}, v_{2 j+2}\right)\right] \\
& =\text { (old }) q_{v_{2 i+1}}-(\text { new }) q_{v_{2 i+1}}+\ell\left(v_{2 i+1}, v_{2 i+2}\right) \\
& =a\left(v_{2 i+1}, v_{2 i+2}\right)+b\left(v_{2 i+1}, v_{2 i+2}\right)-(\text { new }) q_{v_{2 i+1}}-(\text { old }) r_{v_{2 i+2}} \\
& \geq a\left(v_{2 i+1}, v_{2 i+2}\right)-(\text { new }) q_{v_{2 i+1}}+\underline{\pi}\left(v_{2 i+1}, v_{2 i+2}\right),
\end{aligned}
$$

where the last inequality follows from (3.7). The above calculation shows that (c) is true in the same way as in the first iteration. 
We show assertion (d). If (1) $q_{v_{2 i+1}}=a\left(v_{2 i+1}, v_{2 i}\right)+\bar{\pi}\left(v_{2 i+1}, v_{2 i}\right)$ holds, then we can eliminate $\left(v_{2 i+1}, v_{2 i}\right)$ from $B$ by Claim 3.5 and the fact that $q$ does not decrease. If $(2) v_{2 i+1}$ is unsaturated by $x$, then $x$ must be pairwise unstable because of the assertion (b) and Claim 3.3. If (3) $q_{v_{2 i+1}}>a\left(v_{2 i+1}, v_{2 i+2}\right)+b\left(v_{2 i+1}, v_{2 i+2}\right)$, then it follows from Claim 3.3 that $x$ is pairwise unstable.

From the above discussion, if the process goes to Step 1 of Allocation_Stability, then either $B$ can be reduced, or the algorithm may be terminated, unstability having been established. We now show that the same can be said if it does not.

Assume that the process does not go to Step 1 of Allocation_Stability, i.e. none of (1), (2), (3) of (3.8) occurs. After the update (3.8), if we re-calculate arc lengths of all the arcs, $\ell\left(v_{0}, v_{1}\right)$ is equal to the total length $\ell(C)$ of $C$ and all other arcs in $C$ have length 0 by Claim 3.7. By executing (3.8) again for the same cycle $C$ with the re-calculated arc lengths, we can increase $q_{v_{2 i+1}}$ and decrease $r_{v_{2 i+2}}$ by $-\ell(C)$ for $i=0,1, \ldots, k-1$, if neither (1) nor (3) in (3.8) occurs. (Note that (2) cannot occur because we are considering the same cycle $C$.) Thus, if we repeat (3.8), the $q$-values will increase and the $r$-values will decrease by $-\ell(C)$ each, and eventually either (1) or (3) will be satisfied. Since this will require a number of executions of (3.8) in general, we take a shortcut. Define $\delta_{1}, \delta_{2}$ and $L$ by

$$
\begin{aligned}
\delta_{1} & :=\min \left\{a\left(v_{2 i+1}, v_{2 i}\right)+\bar{\pi}\left(v_{2 i+1}, v_{2 i}\right)-q_{v_{2 i+1}} \mid i=0,1, \ldots, k-1\right\} \\
\delta_{2} & :=\min \left\{a\left(v_{2 i+1}, v_{2 i+2}\right)+b\left(v_{2 i+1}, v_{2 i+2}\right)-q_{v_{2 i+1}} \mid i=0,1, \ldots, k-1\right\} \\
L & :=\left\lfloor\min \left\{\delta_{1}, \delta_{2}\right\} /|\ell(C)|\right\rfloor \times|\ell(C)|
\end{aligned}
$$

The value $\delta_{1}$ is the minimum amount for which (1) will occur, likewise $\delta_{2}$ is the minimum amount for which (3) will occur. Thus, neither (1) nor (3) will occur for $\left\lfloor\min \left\{\delta_{1}, \delta_{2}\right\} /|\ell(C)|\right\rfloor$ executions of (3.8). Considering this, we increase $q_{v_{2 i+1}}$ and decrease $r_{v_{2 i+2}}$ by $L$ for $i=$ $0,1, \ldots, k-1$, and if $L<\min \left\{\delta_{1}, \delta_{2}\right\}$, then execute (3.8) again. This will force either (1) or (3) to happen. This is the end of the update in the case where $\widetilde{G}$ has a negative cycle.

We finally deal with the case where $\widetilde{G}$ has no negative cycle. Since $\widetilde{G}$ has no negative cycle, we can define the shortest distance $d(v)$ (with respect to the arc lengths $\ell$ ) of each vertex $v \in P \cup Q$ from $Q$, and construct a shortest path forest $F$ so that for each vertex $v$, the length of the unique path $P(v)$ from the root of the tree including $v$ in $F$ to $v$ is equal to $d(v)$. Since $\ell(i, j) \geq 0$ for all $(i, j) \in X$, we can easily show that if $d(v)<0$ then $d(u) \leq 0$ for all vertices $u \in P(v)$. More strongly, we can construct $F$ so that

for each $v \in P \cup Q$, if $d(v)<0$ then $d(u)<0$ for all internal vertices $u$ of $P(v)$.

We note that there exists a vertex $v$ with $d(v)<0$ by the assumption that the condition of Claim 3.5 is not satisfied. We sort all $\operatorname{arcs}(u, v)$ with $d(v)<0$ in $F$ to satisfy the condition

for each $(u, v)$ with $d(v)<0$, any arc in $P(u)$ appears previous to $(u, v)$.

Let $S=\left(u_{1}, v_{1}\right),\left(u_{2}, v_{2}\right), \ldots,\left(u_{k}, v_{k}\right)$ be such a sorted arc-list. We update $q$ and $r$ according to the list $S$ as follows. 


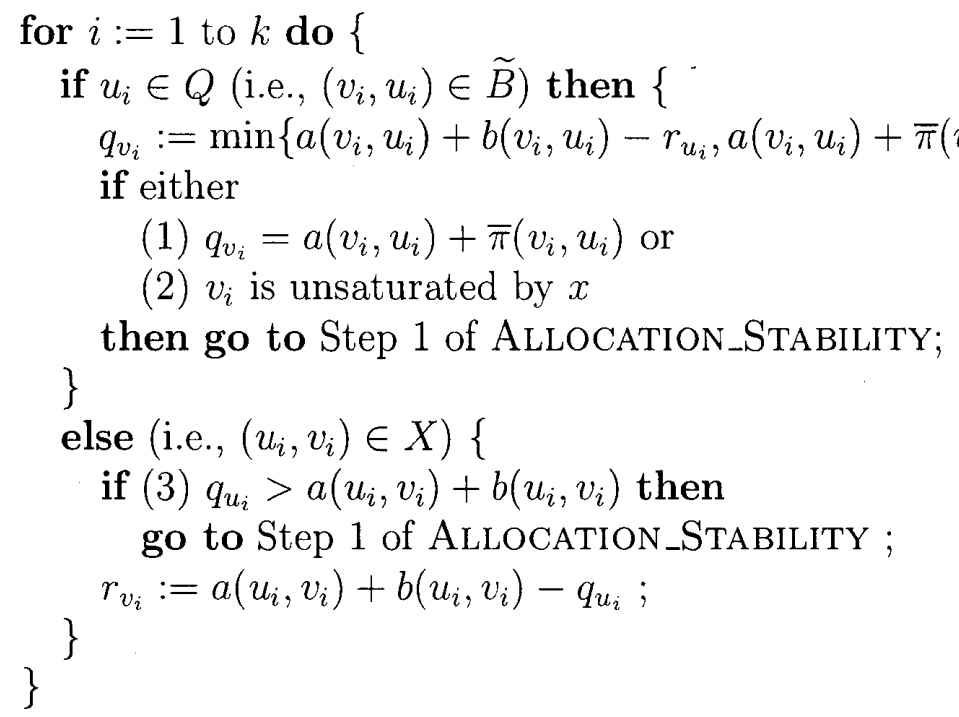

In the same way as the proof of Claim 3.7, we can show the following claim, which implies Claim 3.9.

Claim 3.8. During the update (3.12), we have

(a) the invariants (3.1), (3.5) and (3.6) are preserved,

(b) if neither (1) nor (2) occurs, then $q_{i}$ is increased by $-d(i)$,

(c) if $r_{j}$ is updated then $j$ is saturated by $x$ and $r_{j}$ is decreased by $-d(j)$.

Claim 3.9. If the process goes to Step 1 of AllocATION_STABILITY in (3.12) then either at least one pair can be eliminated from $B$ or $x$ must be pairwise unstable. Otherwise, after the update (3.12), we have $a(i, j)+b(i, j) \geq q_{i}+r_{j}$ for all $(i, j) \in X$, and $a(i, j)+b(i, j) \leq q_{i}+r_{j}$ for all $(i, j) \in \widetilde{B}$.

Proof. The first assertion can be shown in the same manner as the proof of Claim 3.7. We will prove the second assertion. Since the process did not go to Step 1 of AllocATION_STABILITY in (3.12), $q_{v}$ or $r_{v}$ was updated if and only if $d(v)<0$. Thus, Claim 3.8 implies that

$$
\begin{aligned}
(\text { new }) q_{i}-(\text { old }) q_{i} & =\max \{0,-d(i)\} \quad(\forall i \in P), \\
(\text { old }) r_{j}-(\text { new }) r_{j} & =\max \{0,-d(j)\} \quad(=-d(j)) \quad(\forall j \in Q),
\end{aligned}
$$

where $\max \{0,-d(j)\}=-d(j)$ follows from the fact that $j \in Q$ can be a root. For a pair $(i, j) \in X$, we have

$$
\begin{aligned}
& a(i, j)+b(i, j)-(\text { new }) q_{i}-(\text { new }) r_{j} \\
= & a(i, j)+b(i, j)-(\text { old }) q_{i}-\max \{0,-d(i)\}-(\text { old }) r_{j}-d(j) \\
= & \min \{\ell(i, j)-d(j), \ell(i, j)+d(i)-d(j)\} \geq 0
\end{aligned}
$$

where the last equality follows from the facts that $\ell(i, j) \geq 0, d(j) \leq 0$, and the relation on shortest distances $\ell(i, j)+d(i) \geq d(j)$. Similarly, for a pair $(i, j) \in \widetilde{B}$, we have

$$
\begin{aligned}
& (\text { new }) q_{i}+(\text { new }) r_{j}-a(i, j)-b(i, j) \\
= & \ell(j, i)+d(j)-\min \{0, d(i)\} \geq \ell(j, i)+d(j)-d(i) \geq 0,
\end{aligned}
$$

where the last inequality follows from the shortest distance relation. 
Summing up the above discussions, we obtain the following algorithm.

\section{Algorithm Allocation_Stability}

Input: a feasible allocation $x$;

Output: "Yes" if $x$ is pairwise stable; otherwise "No;"

Step 0: $\quad$ set $X:=\{(i, j) \in E \mid x(i, j)=1\}, B:=E \backslash X$, and $q$ by (3.2);

if $b(i, j)<\underline{\pi}(i, j)$ for some $(i, j) \in X$ then output "No" and stop ;

Step 1: calculate $r \in \mathbf{R}^{Q}$ by (3.3) and (3.4) ;

Step 2: for each $(i, j) \in X$ do

if either

(1) $i$ is unsaturated by $x$ and $q_{i}>0$, or

(2) $q_{i}>a(i, j)+\bar{\pi}(i, j)$, or

(3) $q_{i}>a(i, j)+b(i, j)$

then output "No" and stop ;

Step 3: for each $(i, j) \in B$ with $a(i, j)+\bar{\pi}(i, j) \leq q_{i}$ do $B:=B \backslash\{(i, j)\}$;

if $a(i, j)+b(i, j) \leq q_{i}+r_{j}$ or $b(i, j)-\underline{\pi}(i, j) \leq r_{j}$ for each $(i, j) \in B$ then output "Yes" and stop ;

if $B$ was reduced in this step then go to Step 1 ;

Step 4: construct the directed graph $\widetilde{G}$ and calculate the arc lengths $\ell$;

if $\widetilde{G}$ has a negative cycle then \{

find a negative cycle $C=v_{0}, v_{1}, \ldots, v_{2 k}\left(=v_{0}\right)$ satisfying Proposition 3.6 ;

execute (3.8), and then, calculate $\delta_{1}, \delta_{2}$ and $L$ by (3.9) ;

increase $q_{v_{2 i+1}}$ and decrease $r_{v_{2 i+2}}$ by $L$ for $i=0,1, \ldots, k-1$;

\}

if $L<\min \left\{\delta_{1}, \delta_{2}\right\}$ then execute (3.8) again;

else \{

calculate the shortest distances of all vertices of $\widetilde{G}$ from $Q$;

construct a forest $F$ having (3.10) ;

\} construct a list $S=\left(u_{1}, v_{1}\right), \ldots,\left(u_{k}, v_{k}\right)$ having (3.11), and execute (3.12);

go to Step 1.

We consider the correctness of ALLOCATION_STABILITY. The above discussion assumed that any component of $q$ does not decrease and any component of $r$ does not increase during Allocation_Stability. We now verify this.

Claim 3.10. During execution of ALlocation_StABILITY, any component of $q$ does not decrease and any component of $r$ does not increase.

Proof. In Allocation_Stability, $q$ is updated in (3.8) and (3.12). Claims 3.7 and 3.8 guarantee that any component of $q$ does not decrease. On the other hand, $r$ is updated at Step 1 and in (3.8) and (3.12). Claims 3.7 and 3.8 also guarantee that any component of $r$ does not increase in (3.8) and (3.12). Since $q_{i}$ increases or remains the same, $p_{i j}$ also increases or remains the same in (3.3). Hence, $r_{j}$ does not increase by (3.4) at Step 1.

By combining the previous discussion in this subsection and Claim 3.10, we obtain the following result.

Proposition 3.11. If Allocation_Stability stops, it outputs the correct answer.

To show the correctness of ALLOCATION_STABILITY, it is sufficient to show that ALLOCATION_STABILITY terminates in a finite number of iterations. 


\section{Proposition 3.12. Allocation_Stability terminates in $O(|E|)$ iterations.}

Proof. We note that no pair is added into $B$ in steps other than Step 0. We will show the assertion of this proposition by using $B$ and its subset $\widetilde{B}$. When the process moves from Step 3 to Step 1, at least one pair is eliminated from $B$. Thus, this occurs at most $|E|$ times. Next we consider the case where $\widetilde{G}$ has a negative cycle. Claim 3.7 guarantees either the termination of ALLOCATION_STABILITY or the reduction of $B$. Thus, this case occurs at most $|E|$ times. On the other hand, in the case where $\widetilde{G}$ has no negative cycle, Claim 3.9 implies that the case where the process moves to Step 1 from $(3.12)$ occurs at most $|E|$ times. Finally we deal with the case where (3.12) terminates and the process moves to Step 1. At Step 1 in the next iteration, $r$ remains the same because $r_{j} \leq a(i, j)+b(i, j)-q_{i}$ for all $(i, j) \in X$ by Claim 3.9. Thus, if Allocation_Stability does not terminate at Step 3 , then at least one pair $(i, j) \in B \backslash \widetilde{B}$ satisfies $b(i, j)-\underline{\pi}(i, j)>r_{j}$ because Claim 3.9 guarantees that all pairs $(u, v)$ in $\widetilde{B}$ satisfy $a(u, v)+b(u, v) \leq q_{u}+r_{v}$ at this time. Thus, at least one pair was added to $\widetilde{B}$. Since no pair is moved from $\widetilde{B}$ to $B \backslash \widetilde{B}$, the final case also occurs at most $|E|$ times.

The next theorem is a direct consequence of Propositions 3.11 and 3.12.

Theorem 3.13. Given a feasible allocation $x$, AllocAtion_STABILITY outputs "Yes" if $x$ is pairwise stable; otherwise "No."

Obviously, each step of ALLOCATION_STABILITY terminates in polynomial time in the number of agents. Proposition 3.12 implies that Allocation_STABILITY terminates in polynomial time in the number of agents. We can easily check whether a given allocation $x$ is feasible or not. If it is feasible, we can check its pairwise stability in polynomial time in the number of agents by using ALLOCATION_STABILITY. Thus, we obtain our main theorem.

Theorem 3.14. The decision problem of checking the pairwise stability of a given allocation can be solved in polynomial time in the number of agents.

\subsection{Checking pairwise stability of a feasible salary vector}

We now turn to the problem of checking pairwise stability of feasible salary vectors. Unfortunately, unlike the case of allocations, it turns out that this problem is NP-complete, if we restrict the type of stability, to conditions (as1) to (as4) of Theorem 2.1; we say that a feasible salary vector $p$ is a strongly pairwise stable salary vector if there exist a feasible allocation $x, z_{P}$ and $z_{Q}$ satisfying the conditions (as1) to (as4) of Theorem 2.1.

Theorem 3.15. Given a feasible salary vector $p$, the problem of deciding whether $p$ is a strongly pairwise stable salary vector is $N P$-complete.

Proof. It is easy to see that the problem is in NP. We show that it is indeed NP-complete by reducing SAT. Let $U=\left\{u_{1}, u_{2}, \ldots, u_{n}\right\}$ be a set of variables and $\mathcal{C}=\left\{C_{1}, C_{2}, \ldots, C_{m}\right\}$ be a set of clauses specifying an arbitrary instance of SAT. We will construct sets of agents $P, Q$, vectors $\lambda, \mu, a, b$ and $\underline{\pi}, \bar{\pi}$, such that there exists some feasible allocation $x$ such that $(x, \mathbf{0})$ is a pairwise stable outcome satisfying Theorem 2.1 if and only if the instance of SAT is satisfiable, where $\mathbf{0}$ is the vector of all zeros. We define $d_{i}$ (respectively $\bar{d}_{i}$ ) as the number of clauses which contain the literal $u_{i}$ (respectively $\overline{u_{i}}$ ). However, for the sake of simplicity, we will use $d_{i}$ to denote the number of clauses which contain the literal $y_{i}$, where $y_{i}$ is either $u_{i}$ or $\overline{u_{i}}$.

Let $P=\left\{u_{1}, \overline{u_{1}}, \ldots, u_{n}, \overline{u_{n}}\right\}$, that is $P$ has $2 n$ agents corresponding to each literal. The set $Q$ consists of one agent for each clause, one agent for each variable, and $d_{i}+1$ "dummy" 
agents for each literal $y_{i}$ :

$$
Q=\left\{C_{1}, \ldots, C_{m}\right\} \cup\{1, \ldots, n\} \cup\left\{D_{1,1}, \ldots, D_{1, d_{1}+1}\right\} \cup \cdots \cup\left\{\bar{D}_{n, 1}, \ldots, \bar{D}_{n, \bar{d}_{n}+1}\right\},
$$

the sets $\left\{D_{i, 1}, \ldots, D_{i, d_{i}+1}\right\}$ and $\left\{\bar{D}_{i, 1}, \ldots, \bar{D}_{i, \bar{d}_{i+1}}\right\}$ being the dummy agents for literals $u_{i}$ and $\overline{u_{i}}$, respectively. The vector $\lambda$ is defined by $\lambda\left(y_{i}\right)=d_{i}+1$ for each literal $y_{i}$, and $\mu$ is set to be the vector of all 1s. In the rest of the proof we will refer to elements of $E=P \times Q$ as edges. We now define $a, b, \underline{\pi}$ and $\bar{\pi}$.

For literal $y_{i}$ and clause $C_{j}$ with $y_{i} \in C_{j}$, set

$$
a\left(y_{i}, C_{j}\right)=0, \quad b\left(y_{i}, C_{j}\right)=1, \quad \underline{\pi}\left(y_{i}, C_{j}\right)=-\infty, \quad \bar{\pi}\left(y_{i}, C_{j}\right)=+\infty .
$$

We will call these type 1 edges. For literals $u_{i}, \overline{u_{i}}$ and $i \in Q$

$$
\begin{aligned}
& a\left(u_{i}, i\right)=1, \quad b\left(u_{i}, i\right)=1, \quad \underline{\pi}\left(u_{i}, i\right)=-\infty, \quad \bar{\pi}\left(u_{i}, i\right)=+\infty, \\
& a\left(\overline{u_{i}}, i\right)=1, \quad b\left(\overline{u_{i}}, i\right)=1, \quad \underline{\pi}\left(\overline{u_{i}}, i\right)=-\infty, \quad \bar{\pi}\left(\overline{u_{i}}, i\right)=+\infty,
\end{aligned}
$$

these are called type2 edges. For literal $y_{i}$ and dummy agent $D_{i, k},\left(k=1,2, \ldots, d_{i}+1\right)$ let

$$
a\left(y_{i}, D_{i, k}\right)=1, b\left(y_{i}, D_{i, k}\right)=0, \underline{\pi}\left(y_{i}, D_{i, k}\right)=0, \bar{\pi}\left(y_{i}, D_{i, k}\right)=+\infty .
$$

We name these type3 edges. Finally, for all other edges $(i, j) \in E$ we set

$$
a(i, j)=b(i, j)=-1, \underline{\pi}=-\infty, \bar{\pi}=+\infty .
$$

Note that for these edges, $x(i, j)$ must be equal to 0 in any pairwise stable outcome $(x, p)$.

Clearly, $p=\mathbf{0}$ is a feasible salary vector. We now prove that there exists a feasible allocation $x$ and vectors $z_{P}, z_{Q}$ satisfying conditions (as1) to (as4) if and only if the given instance of SAT is satisfiable.

First suppose that the instance of SAT is satisfiable, and consider any truth assignment which satisfies it. The $x-$ values are determined as follows. For each variable $u_{i}$, if $u_{i}$ is true, then set $x\left(u_{i}, i\right)=1$, otherwise set $x\left(\overline{u_{i}}, i\right)=1$. For each clause $C_{j}$, choose an arbitrary true literal $y_{i} \in C_{j}$, and set $x\left(y_{i}, C_{j}\right)=1$. At this point, for any literal $y_{i}$, the number of edges $\left(y_{i}, k\right)$ with $x$-value equal to one is at most $d_{i}+1$. Now choose the appropriate number of dummy agents to bring the total to exactly $d_{i}+1$ and set $x\left(y_{i}, D_{i, k}\right)=1$ for all of them. All other edges have $x$-values set to zero. As for $z_{P}$ and $z_{Q}$, all values are set to one except for the type3 edges with $x\left(y_{i}, D_{i, k}\right)=0$; for these edges, we set $z_{P}\left(y_{i}, D_{i, k}\right)=0$. It is easy to see that such $x, z_{P}$ and $z_{Q}$ satisfy conditions (as1) to (as4).

Conversely, suppose that $x, z_{P}, z_{Q}$ together with $p=\mathbf{0}$ satisfy (as1) to (as4). Conditions (as3) and (as4) imply that

$$
z_{P}(i, j)=z_{Q}(i, j)=1
$$

for all type1 and type2 edges $(i, j)$, thus for condition (as2) to hold for $i \in Q$, exactly one of $x\left(u_{i}, i\right)$ and $x\left(\overline{u_{i}}, i\right)$ must be equal to one. This corresponds to setting one of $u_{i}$ and $\overline{u_{i}}$ to be true. For simplicity, we will abuse terminology, and henceforth say that any literal $y_{i}\left(=u_{i}\right.$ or $\left.\overline{u_{i}}\right)$ with $x\left(y_{i}, i\right)=1$ is true. Now consider a literal $y_{i}$ which is false, i.e., $x\left(y_{i}, i\right)=0$. Because $a\left(y_{i}, i\right)=1, z_{P}\left(y_{i}, i\right)=1$, and condition (as1), there must be exactly $d_{i}+1$ edges with $x\left(y_{i}, k\right)=1$ such that $a\left(y_{i}, k\right)=1$. Since the $a$-values of all type 1 edges are zero, any typel edge $\left(y_{i}, C_{j}\right)$ with $x\left(y_{i}, C_{j}\right)=1$ must have $y_{i}$ true. But since the $z_{Q}$ and $b$ values are one for all typel edges, there is always exactly one such edge for any clause $C_{j}$, completing the proof. 


\section{Structures of Pairwise Stable Outcomes}

In this section, we consider problems concerning the structures of pairwise stable outcomes. Since our model contains the assignment model and stable marriage model as special cases, it is natural to ask whether the structures of those cases carry over to ours. We will consider four possibilities.

The first is the existence of a common strongly stable pairwise salary vector. For the assignment case (i.e., the case where $\underline{\pi}=(-\infty, \ldots,-\infty)$ and $\bar{\pi}=(+\infty, \ldots,+\infty)$ ), it follows from the duality of linear programming that there exists a feasible salary vector $p$ such that for any pairwise stable allocation $x,(x, p)$ satisfies the conditions in Theorem 2.1. On the other hand, for the marriage case where $\underline{\pi}=\bar{\pi}=\mathbf{0}$, the above property trivially holds. Thus, one may have the following question for our model.

Question 1. Does there exist a feasible salary vector $p$ such that for all pairwise stable allocations $x$, the outcome $(x, p)$ satisfies the conditions in Theorem 2.1?

We have a negative answer to Question 1.

Example 1. Consider the instance where

$$
\begin{aligned}
& P=\{1,2,3\}, \quad Q=\left\{1^{\prime}, 2^{\prime}, 3^{\prime}\right\}, \\
& \lambda(i)=1(\forall i \in P), \quad \mu(j)=1(\forall j \in Q), \\
& \left(a_{11^{\prime}}, a_{12^{\prime}}, a_{13^{\prime}}, a_{21^{\prime}}, a_{22^{\prime}}, a_{23^{\prime}}, a_{31^{\prime}}, a_{32^{\prime}}, a_{33^{\prime}}\right)=(-1,1,3,2,3,1,1,2,3), \\
& \left(b_{11^{\prime}}, b_{12^{\prime}}, b_{13^{\prime}}, b_{21^{\prime}}, b_{22^{\prime}}, b_{23^{\prime}}, b_{31^{\prime}}, b_{32^{\prime}}, b_{33^{\prime}}\right)=(-1,2,1,2,1,3,3,3,2), \\
& \underline{\pi}\left(3,1^{\prime}\right)=-\infty, \quad \underline{\pi}(i, j)=0\left(\forall(i, j) \in E \backslash\left\{\left(3,1^{\prime}\right)\right\}\right), \\
& \bar{\pi}\left(3,1^{\prime}\right)=+\infty, \quad \bar{\pi}(i, j)=0\left(\forall(i, j) \in E \backslash\left\{\left(3,1^{\prime}\right)\right\}\right) .
\end{aligned}
$$

For this instance, there exist three pairwise stable allocations $X^{1}=\left\{\left(1,2^{\prime}\right),\left(2,1^{\prime}\right),\left(3,3^{\prime}\right)\right\}$, $X^{2}=\left\{\left(1,2^{\prime}\right),\left(2,3^{\prime}\right),\left(3,1^{\prime}\right)\right\}$, and $X^{3}=\left\{\left(1,3^{\prime}\right),\left(2,2^{\prime}\right),\left(3,1^{\prime}\right)\right\}$. For $X^{1}, X^{2}$, and $X^{3}$, the conditions in Theorem 2.1 hold only if $1 \leq p\left(3,1^{\prime}\right) \leq 2, p\left(3,1^{\prime}\right)=1$, and $2 \leq p\left(3,1^{\prime}\right) \leq 3$, respectively. Hence, this instance gives a counterexample to Question 1.

The next possibility is the lattice structure of stable salary vectors when the allocation is fixed. By lattice structure, we mean that if $p^{1}$ and $p^{2}$ are in a specified set, $p^{1} \wedge p^{2}$ and $p^{1} \vee p^{2}$ are also contained in that set, where $\left(p^{1} \wedge p^{2}\right)(e)=\min \left\{p^{1}(e), p^{2}(e)\right\}$ and $\left(p^{1} \vee p^{2}\right)(e)=\max \left\{p^{1}(e), p^{2}(e)\right\}$ for all $e \in E$. For this, we have an affirmative answer; for a fixed pairwise stable allocation $x$, the set of feasible salary vectors $p$ such that $(x, p)$ satisfies the conditions in Theorem 2.1 has a lattice structure. More precisely, the next proposition holds.

Proposition 4.1. For a fixed pairwise stable allocation $x$, if $\left(x, p^{1}, z_{P}^{1}, z_{Q}^{1}\right)$ and $\left(x, p^{2}, z_{P}^{2}, z_{Q}^{2}\right)$ satisfy the conditions in Theorem 2.1, then $\left(x, p^{1} \wedge p^{2}, z_{P}^{1} \wedge z_{P}^{2}, z_{Q}^{1} \vee z_{Q}^{2}\right)$ and $\left(x, p^{1} \vee p^{2}, z_{P}^{1} \vee\right.$ $\left.z_{P}^{2}, z_{Q}^{1} \wedge z_{Q}^{2}\right)$ also satisfy these conditions.

Proof. Here we show that $\left(x, p, z_{P}, z_{Q}\right)=\left(x, p^{1} \wedge p^{2}, z_{P}^{1} \wedge z_{P}^{2}, z_{Q}^{1} \vee z_{Q}^{2}\right)$ satisfies (as1), (as2), (as3) and (as4).

If $z_{P}(i, j)=0$ then we have $z_{P}^{1}(i, j)=0$ or $z_{P}^{2}(i, j)=0$, which implies (as3) for $\left(x, p, z_{P}, z_{Q}\right)$. If $z_{Q}(i, j)=0$ then we have $z_{Q}^{1}(i, j)=z_{Q}^{2}(i, j)=0$, which implies (as4) for $\left(x, p, z_{P}, z_{Q}\right)$.

Next we show that $\left(x, p, z_{P}, z_{Q}\right)$ satisfies (as1). Since $a(i, j)+p^{1}(i, j) \geq 0$ and $a(i, j)+$ $p^{2}(i, j) \geq 0$ for all $(i, j) \in X$, we have $a(i, j)+p(i, j) \geq 0$ for all $(i, j) \in X$. If $i$ is unsaturated by $x$ then $a(i, k)+p^{1}(i, k) \leq 0$ for all $(i, k) \notin X$ with $z_{P}^{1}(i, k)=1$, and $a(i, k)+p^{2}(i, k) \leq 0$ for all $(i, k) \notin X$ with $z_{P}^{2}(i, k)=1$, which imply $a(i, k)+p(i, k) \leq 0$ for all $(i, k) \notin X$ with 
$z_{P}(i, k)=1$. If $i$ is saturated by $x$ then $a(i, j)+p^{1}(i, j) \geq a(i, k)+p^{1}(i, k)$ for all $(i, j) \in X$ and for all $(i, k) \notin X$ with $z_{P}^{1}(i, k)=1$, and $a(i, j)+p^{2}(i, j) \geq a(i, k)+p^{2}(i, k)$ for all $(i, j) \in X$ and for all $(i, k) \notin X$ with $z_{P}^{2}(i, k)=1$. Thus, we have

$$
\begin{aligned}
a(i, j)+p(i, j) & =\min \left\{a(i, j)+p^{1}(i, j), a(i, j)+p^{2}(i, j)\right\} \\
& \geq \min \left\{a(i, k)+p^{1}(i, k), a(i, k)+p^{2}(i, k)\right\} \\
& =a(i, k)+p(i, k)
\end{aligned}
$$

for all $(i, j) \in X$ and for all $(i, k) \notin X$ with $z_{P}(i, k)=1$. The above discussion verifies that $\left(x, p, z_{P}, z_{Q}\right)$ satisfies (as1).

Finally we show that $\left(x, p, z_{P}, z_{Q}\right)$ satisfies (as2). In the same way as above, we can show that 1) $b(i, j)-p(i, j) \geq 0$ for all $(i, j) \in X$; 2$)$ if $i$ is unsaturated by $x$ then $b(i, k)-p(i, k) \leq$ 0 for all $(i, k) \notin X$ with $\left.z_{Q}(i, k)=1 ; 3\right)$ if $i$ is saturated by $x$ then $b(i, j)-p(i, j) \geq$ $b(i, k)-p(i, k)$ for all $(i, j) \in X$ and for all $(i, k) \notin X$ with $z_{P}(i, k)=1$. This implies that $\left(x, p, z_{P}, z_{Q}\right)$ satisfies (as2).

Now, what happens when we allow the allocation $x$ to be arbitrary? For the assignment case, it is known that the set of feasible salary vectors $p$ such that $(x, p)$ satisfies the conditions in Theorem 2.1 for some pairwise stable allocation $x$ forms a lattice structure [3]. Question 2. Does the set of feasible salary vectors $p$ :

$$
\{p \mid(x, p) \text { satisfies the conditions in Theorem } 2.1 \text { for some } x\}
$$

form a lattice structure?

The answer is negative.

Example 2. Consider the instance where

$$
\begin{aligned}
& P=\{1,2\}, \quad Q=\left\{1^{\prime}, 2^{\prime}\right\}, \\
& \lambda(i)=1(\forall i \in P), \quad \mu(j)=1(\forall j \in Q), \\
& \left(a_{11^{\prime}}, a_{12^{\prime}}, a_{21^{\prime}}, a_{22^{\prime}}\right)=(0,0,-1,-1), \\
& \left(b_{11^{\prime}}, b_{12^{\prime}}, b_{21^{\prime}}, b_{22^{\prime}}\right)=(4,4,3,3), \\
& \underline{\pi}(i, j)=0(\forall(i, j) \in E), \\
& \bar{\pi}(1, j)=0, \quad \bar{\pi}(2, j)=2(\forall j \in Q) .
\end{aligned}
$$

For a feasible salary vector $p^{1}$ with $p^{1}\left(2,1^{\prime}\right)=0$ and $p^{1}\left(2,2^{\prime}\right)=2, X^{1}=\left\{\left(1,1^{\prime}\right),\left(2,2^{\prime}\right)\right\}$ together with $p^{1}$ satisfies the conditions in Theorem 2.1 by setting $z_{Q}^{1}\left(1,2^{\prime}\right)=0$. On the other hand, for a feasible salary vector $p^{2}$ with $p^{2}\left(2,1^{\prime}\right)=2$ and $p^{2}\left(2,2^{\prime}\right)=0, X^{2}=\left\{\left(1,2^{\prime}\right),\left(2,1^{\prime}\right)\right\}$ together with $p^{2}$ satisfies the conditions in Theorem 2.1 by setting $z_{Q}^{2}\left(1,1^{\prime}\right)=0$. However, $p=p^{1} \wedge p^{2}=\mathbf{0}$ yields no pairwise stable allocation, because $a\left(2,1^{\prime}\right)+p\left(2,1^{\prime}\right)=a\left(2,2^{\prime}\right)+$ $p\left(2,2^{\prime}\right)=-1$. This gives a counterexample to Question 2.

Finally, we deal with the connectivity of the set of feasible salary vectors satisfying the conditions in Theorem 2.1. For the assignment case, the set of feasible salary vectors $p$ such that for some pairwise stable allocation $x,(x, p)$ satisfies the conditions in Theorem 2.1 is connected. That is, if $\left(x, p^{1}\right)$ and $\left(x, p^{2}\right)$ satisfy the conditions in Theorem 2.1 , then there exists a continuous mapping $\varphi:[0,1] \rightarrow \mathbf{R}^{E}$ such that $\varphi(0)=p^{1}, \varphi(1)=p^{2}$ and $(x, \varphi(t))$ satisfies the conditions in Theorem 2.1 for all $t \in[0,1]$.

Question 3. Let $x$ be a pairwise stable allocation. Does the set of feasible salary vectors $p$ such that $(x, p)$ satisfies the conditions in Theorem 2.1 is connected? 
The answer to this question is also negative.

Example 3. Consider the instance with

$$
\begin{aligned}
& P=\{1,2\}, \quad Q=\left\{1^{\prime}, 2^{\prime}\right\}, \\
& \lambda(i)=1(\forall i \in P), \quad \mu(j)=1(\forall j \in Q), \\
& \left(a_{11^{\prime}}, a_{12^{\prime}}, a_{21^{\prime}}, a_{22^{\prime}}\right)=(2,2,3,2), \\
& \left(b_{11^{\prime}}, b_{12^{\prime}}, b_{21^{\prime}}, b_{22^{\prime}}\right)=(1,0,3,2) \\
& -2 \leq p\left(1,1^{\prime}\right) \leq 1, \quad-1 \leq p\left(1,2^{\prime}\right) \leq 0 \\
& 0 \leq p\left(2,1^{\prime}\right) \leq 1, \quad-2 \leq p\left(2,2^{\prime}\right) \leq 2
\end{aligned}
$$

We consider a feasible allocation $X=\left\{\left(1,1^{\prime}\right),\left(2,2^{\prime}\right)\right\}$. By setting

$$
p\left(1,1^{\prime}\right)=-2, \quad p\left(1,2^{\prime}\right)=-1, \quad p\left(2,1^{\prime}\right)=0, \quad p\left(2,2^{\prime}\right)=1,
$$

$(X, p)$ satisfies the conditions in Theorem 2.1. On the other hand, by setting

$$
p\left(1,1^{\prime}\right)=1, \quad p\left(1,2^{\prime}\right)=0, \quad p\left(2,1^{\prime}\right)=1, \quad p\left(2,2^{\prime}\right)=2,
$$

$X$ also satisfies these conditions. However, we can show that $X$ cannot satisfy these conditions if $0<p\left(2,1^{\prime}\right)<1$.

Acknowledgements The authors would like to express their gratitude to the anonymous referees for their helpful suggestions which greatly improved the presentation of this paper.

\section{References}

[1] K. Eriksson and J. Karlander: Stable matching in a common generalization of the marriage and assignment models. Discrete Mathematics, 217 (2000), 135-156.

[2] S. Fujishige and A. Tamura: A general two-sided matching market with discrete concave utility functions. Discrete Applied Mathematics, 154 (2006), 950-970.

[3] S. Fujishige and A. Tamura: A two-sided discrete-concave market with bounded side payments: An approach by discrete convex analysis. Mathematics of Operations Research, 32 (2007), 136-155.

[4] D. Gale and L.S. Shapley: College admissions and the stability of marriage. The American Mathematical Monthly, 69 (1962), 9-15.

[5] M. Kaneko: The central assignment game and the assignment markets. Journal of Mathematical Economics, 10 (1982), 205-232.

[6] K. Murota: Convexity and Steinitz's exchange property. Advances in Mathematics, 124 (1996), 272-311.

[7] K. Murota: Discrete convex analysis. Mathematical Programming, 83 (1998), 313-371.

[8] K. Murota: Discrete Convex Analysis (Society for Industrial and Applied Mathematics, Philadelphia, 2003).

[9] A.E. Roth and M.A.O. Sotomayor: Stable outcomes in discrete and continuous models of two-sided matching: A unified treatment. Revita de Econometria, 16 (1996), 1-24.

[10] L.S. Shapley and M. Shubik: The assignment game I: The core. International Journal of Game Theory, 1 (1972), 111-130.

[11] M. Sotomayor: Existence of stable outcomes and the lattice property for a unified matching market. Mathematical Social Sciences, 39 (2000), 119-132. 
Akihisa Tamura

Department of Mathematics

Keio University

3-14-1 Hiyoshi, Kouhoku-ku, Yokohama-shi, Kanagawa 223-8522, Japan

E-mail: aki-tamura@math.keio.ac.jp 